\title{
Toxicity Grade Scale Type Terminology
}

National Cancer Institute

\section{Source}

National Cancer Institute. Toxicity Grade Scale Type Terminology. NCI Thesaurus. Code C105753.

Terminology codelist containing values associated with identifiable classes of grading scales used to classify toxicity. 